\title{
Médecin, politicien et liberté... je t'aime, moi non plus
}

\section{Fabienne Gay-Crosier}

Dr, spécialiste en Allergologie Immunologie clinique, Médecine interne

\section{Réflexion au sujet du secret médical, du rôle du médecin face à la politique et à la liberté: où se situe le libéralisme en médecine?}

La profession médicale ne limite pas le droit de penser. Ceci fait partie des droits humains fondamentaux. En Suisse, la politique est effectuée par des miliciens; elle permet la multiplicité des idées.

La médecine thérapeutique n'a rien de commun avec la politique. Cependant, comme pour l'approvisionnement en eau, électricité, poste, etc., les gouver-

\section{La profession de médecin a donc besoin} sans aucun doute possible des politiciens. Elle a besoin de concepteurs de produits.

nements doivent assurer un cadre permettant aux intervenants de pouvoir exercer leur rôle et prendre leurs responsabilités. Permettre un cadre logistique de fourniture de produits de qualité pour la médecine diagnostique et thérapeutique en adéquation avec les besoins ne signifie pas infantiliser les intervenants. L'entière responsabilité de l'action diagnostique et thérapeutique en revient aux médecins. Pour le médecin, cela signifie donc de lui permettre de corriger la maladie mais aussi les inégalités issues de notre origine: il se met ainsi en accord avec le serment d'Hippocrate et le respect des droits fondamentaux. Le diagnostic est le fruit du travail intellectuel du médecin. Il est sa propriété au même titre et avec les mêmes avantages que donnent la possession d'une propriété intellectuelle, utilisée pour bénéficier d'un traitement. La maladie, elle, appartient au malade. Le citoyen a voté en Suisse le libre choix du médecin. La demande d'une relation thérapeutique est donc choisie par le patient. Le médecin doit pouvoir choisir également librement de la lui accorder. Accepter ou refuser un mandat fait partie des droits humains les plus fondamentaux. En découle ainsi un choix de mandat, un mandat de confiance dans lequel s'inscrit la relation thérapeutique et où se situe le secret médical. Le secret médical contient à la fois le diagnostic, la maladie et la relation thérapeu- tique: de ce fait, le secret médical est propriété à la fois du médecin et du patient.

Le législateur a voulu laisser une grande liberté d'action au médecin: qualité, efficacité, adéquation au but du traitement et à l'intérêt du patient.

Où se situe donc le libéralisme de la médecine? Il se base sur la liberté de penser et d'agir du médecin qui utilise ses connaissances apprises à l'Université et celles qu'il acquiert par la suite sous sa responsabilité propre: le médecin est en droit de penser librement. Il peut donc selon le mandat que lui donne le patient, appliquer seul la pertinence de ses connaissances et ceci, sans ingérence ni de l'état, ni des assureurs, ni des producteurs de moyens médicaux.

Le médecin reçoit donc la confiance de son patient de «penser pour lui». Penser librement pour un médecin va dès lors signifier avoir accepté de mettre son patient malade au centre de ses préoccupations: il va exercer en pratique quotidienne le serment d'Hippocrate, vieux de deux millénaires et demi. Il va donc observer et étudier son patient, lui offrir la pertinence de ses réflexions en créant, modifiant, adaptant librement les outils du marché à l'individualité de son patient. Cette liberté s'exerce sous sa responsabilité propre et selon le souhait de son patient. Dans l'exercice de cette liberté, le médecin va respecter les droits de son patient dont le premier sera de lui faire recouvrer au plus vite et au mieux sa propre liberté. Le patient a acquis dans cette relation, un droit «positif» à la santé et donc à la liberté. Il n'est donc pas admissible que soient utilisés à mauvais es-

\section{Le législateur a voulu laisser une grande} liberté d'action au médecin: qualité, efficacité, adéquation au but du traitement.

cient ou de façon pervertie, des concepts éthiques et moraux se rapportant à des textes fondamentaux tels que ceux des «Droits de l'Homme», exprimant 


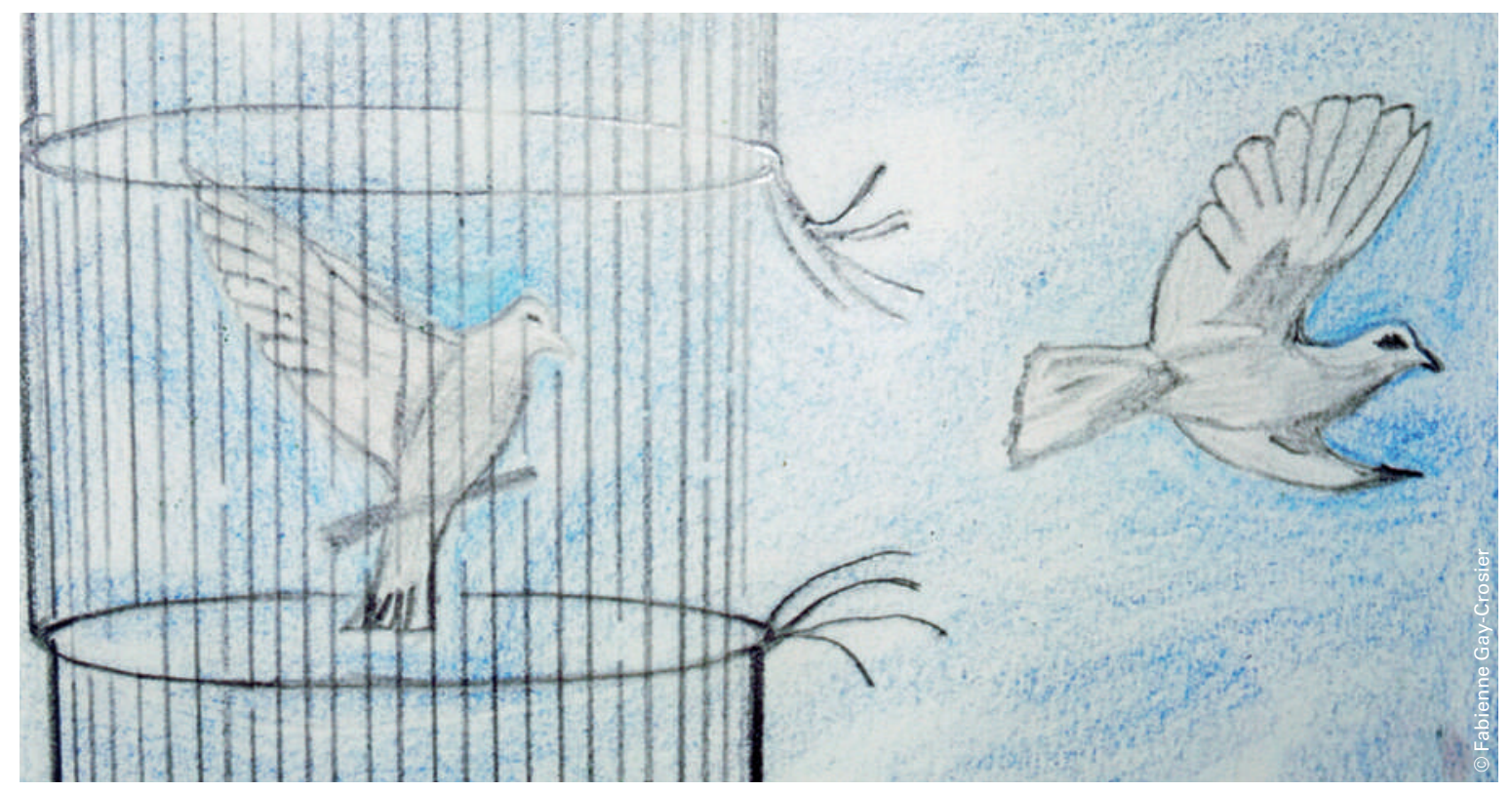

Le médecin est en droit de penser librement, sans ingérence ni de l'état, ni des assureurs, ni des producteurs de moyens médicaux.

des valeurs fondamentales de nos sociétés occidentales que sont la liberté individuelle, le droit à la propriété dont découle entre autre le sens de la responsabilité. Le patient et le médecin n'ont donc pas besoin de règlements bureaucratiques en sus s'immisçant dans leur relation. Cette liberté du médecin, pour son exercice, nécessite cependant pour être efficace, un cadre adéquat permettant la fourniture de produits: une bonne médecine conduit souvent à des succès économiques.

Le travail du médecin en Suisse s'inscrit le plus souvent dans la Loi sur l'assurance maladie. Toute loi implique un contrôle de l'application de la loi. Contrôler le travail du médecin selon cette loi, en respectant le droit des patients, ne peut se faire qu'en tenant compte des diagnostics d'abord et de l'efficience des traitements ensuite. L'adéquation du traitement implique l'adéquation au mandat de la relation thérapeutique entre le médecin et son patient. Tout autre schéma conduit et a conduit à des déviances: ces déviances se font au détriment des malades pour qui cette loi a été édictée.

La profession de médecin a donc besoin sans aucun doute possible des politiciens. Elle a besoin de concep- teurs de produits. Mais la politique se fonde sur des faits. Elle se fait donc avec des gens qui savent. Et ceux qui savent sont ceux qui pensent et qui pratiquent, notamment les praticiens de la relation thérapeutique.

\section{Les médecins libéraux encouragent les} politiciens, les concepteurs et les contrôleurs à questionner les bons interlocuteurs.

Les médecins libéraux encouragent dès lors les politiciens, les concepteurs et les contrôleurs à questionner les bons interlocuteurs. Dans quel but? Echanger activement avec eux, leur permettant ainsi une réflexion, des questions efficientes, adéquates et économiques de leur temps ainsi qu'une action respectueuse et responsable.

\section{Référence}

Adapté avec l'aimable soutien du Dr Henri Siegenthaler, l'auteur de l'article: Médecine libérale ou médecine sociale ou socialiste. Bull Méd Suisses. 2011;92(45):1757-9. 